\title{
28 Resarch Soure \\ Psychological, social, and motivational factors in the drug user care-seekers
}

\section{Sana Shahrabadi}

Gorgan Islamic Azad University

Amir Jalali ( $\nabla$ jalali_amir@yahoo.com )

Kermanshah University of Medical Sciences https://orcid.org/0000-0002-0307-879X

\section{Rostam Jalali}

Kermanshah University of Medical Sciences

\author{
Ali Gholami \\ Kermanshah University of Medical Sciences
}

\section{Research}

Keywords: Psychosocial performance, motivation for treatment, drug users, demographical variables

Posted Date: January 31st, 2020

DOI: https://doi.org/10.21203/rs.2.22403/v1

License: (c) (i) This work is licensed under a Creative Commons Attribution 4.0 International License. Read Full License 


\section{Abstract}

Background In addition to the family and society's support, the drug user plays a key role in the process of treatment and rehabilitation. Therefore, it is imperative to find the psychological, social, and motivational factors that can help them in the treatment process. The present study is an attempt to determine the relationship of psychological, social, and motivational factors and demographics of drug user care-seekers.

Methods An analytical cross-sectional study was carried out. Psychological, Social, and Motivational Performance Questionnaire for drug users was first translated into Farsi and validated after securing a permission from the copyright holder of the tool. Participants were 250 drug user care-seekers under methadone therapy who were selected through convenient sampling. Before analyzing the collected data, validity and reliability of the tool were confirmed using confirmatory and exploratory factor analyses. Given the scale of demographical data, suitable statistics were used to analyze the relationship between demographical variables and psychological, social, and motivational factors.

Results The results of exploratory and confirmatory factor analyses showed that out of 83 statements in the original questionnaire, 55 statements categorized into 11 aspects were usable for Iranian population. The results showed that gender, income, and marital status were effective in psychological performance of the drug users $(P<0.05)$. However, education level, domicile, and type of drug and consumption did not have a significant relationship with social performance of the participants $(p>0.05)$. There was a significant relationship between age, number of children, and history of using drug and psychological performance of the participants $(P<0.01)$. The results showed that the demographics did not have a notable effect on the participants' motivation for treatment; only marital status had a significant relationship with the participants' readiness for treatment $(P<0.05)$.

Conclusion As the results showed, the demographical variables could affect physical, psychological, and motivational readiness in the participants.

\section{Background}

Drug dependence is one of the main psychosocial challenges in today society all around the world (1). Every day, a large number of individuals are lured into drugs (2). According to the World Health Organization, about $5.6 \%$ of $15-64$ years old population in the world have used drugs at least once in their lives. About 31 million in the world suffer the disorders caused by using drugs (3). In the case of Iran, the youth are at a high risk of developing drugs dependence due to cultural reasons, wrong beliefs, and neighboring one of the main producer countries of narcotic substances (4). Immense losses in form of lost lives and financial resources and the social outcomes (heavy costs, death, suicide, crime, divorce, sexually transmitted diseases like HIV and hepatitis) caused by using drugs are not negligible (5). Shahbazi et al. (2017) reported that mortality rate of drug addiction in Iran was 38.4 individuals per one million, which is higher than the world average rate(6). 
Abusive use of drugs in psychological patients is a prevalent issue that affects social and occupational performance of the individual (7). Studies on abusive use of drug have shown that there is a direct relationship between abusive use of drugs and mental health (8). Developing drug dependence hinders fulfillment of social, spiritual, and emotional roles of the user at social and family levels; which causes problems for the society and family (9). Psychosocial performance is a key factor in the treatment and rehabilitation of drug user care-seeker (10-12). Personality factors and psychological ones in particular like happiness and self-confidence are of the main factors in the decision to quit (13).

Social, psychological, and motivational factors can help the care-seekers in making decision to quit drugs (12). Physical, psychological, and motivational factors can help the care-seekers in the treatment process and afterwards. In addition, demographical factors can be also effective (8). As suggested by studies, variables age, gender, marital status, and education level are effective in psychological performance of drug users (14). In addition, more than one half of the abusive users of drugs suffer psychological disorders (15). On the other hand, perceiving the motivations of drug users is very important for the treatment as it is knowing about the motivational factors when they want to quit (16). Motivation has long been considered as a key factor in the treatment of risk behaviors like abusive use of alcoholic drinks and drugs. It is also highly important in the successful treatment of drugs abusive use $(16,17)$. As showed by studies, there are very important motivational factors in the treatment and rehabilitation of drug user care-seekers $(8,18)$.

Given this introduction, it is essential to comprehend the relationship of demographical variables in drug users and their psychological, social, and motivational performance. Having a deep insight into the effective factors we can take more effective steps to alleviate the damages caused by abusive use of drugs through making more effective decisions to treat the patients. Therefore, the present study is an attempt to survey the relationship between psychological, social, and motivational factors and some of the demographical factors.

\section{Methods}

\section{Study population and sampling}

A cross-sectional and descriptive-analytical study was carried out. At first, Psychological, Social, and Motivational Performance Questionnaire for drug users was translated into Farsi through forwardbackward method (Wild et al. (19)). The tool was translated into Farsi by two independent translators and the translations were checked by a translation team to spot inconsistencies between the two translations. The draft translation was translated back into English by two translators and inconsistencies between the original and translated versions were examined. The draft was designed and arranged as a standard questionnaire and provided to drug user care-seekers to comment on its understandability and clarity. The patients' feedbacks were implemented on the questionnaire and vague and unfamiliar terms were corrected. Afterwards, content validity index (CVI), content validity ratio (CVR), and Kappa coefficient were obtained for the questionnaire. Then, data gathering process was started. 
The study population was all the drug users visiting drug addiction clinics located in Kermanshah CityIran. The participants were selected based on inclusion criteria. The participants were selected through convenient sampling. At first, the participants were briefed about the questionnaire and how to fill it and they signed a written letter of consent. Inclusion criteria were desire to participate, using herbal narcotic drugs, and at least two weeks under methadone therapy. In the tool validation stage, 10 experts gave their opinions about content validity of the tool, and to examine construct validity, the tool was provided to 250 care seekers. Mean age of the participants was $39.24 \pm 11.73$ and mean history of using drugs was 13.8 \pm 11.04 . Totally, $86.6 \%$ were men, $54.8 \%$ were married, $43.6 \%$ did not have a high school diploma, $40.4 \%$ had a high school diploma, and $91.6 \%$ lived in city. To examine content validity, Waltz and Bausell's content validity index (CVI) was used and to examine correlation between the scores of tests and tools (test-retest reliability) Pearson's correlation coefficient was used. In addition, Cronbach's alpha was employed to check internal consistency of the tool. Exploratory and confirmatory factor analyses were used to check the construct validity. The relationship between demographical variables and psychological, social, and motivational performance was examined using suitable statistics.

\section{Instrument}

A demographics form and Psychological, Social, and Motivational Performance Questionnaire (20) was used. The questionnaire is comprised of 83 statements and 11 categories viz. self-esteem, depression, anxiety, confidence in decision making (psychological performance aspect); childhood problems, violence, risk taking attitude, and social adaptability (social performance aspect); realizing problem, tendency to seek help from others, and readiness for treatment (treatment motivation aspect).

\section{Findings}

\section{Validation of the tool}

The first step to check validity of the tool is content validity check. Waltz and Bausell's CVI was employed to this end. As the results showed, CVI and CVR were acceptable for all the statements and no statement was omitted at this stage. To examine reliability of the tool, test-retest technique was used through Pearson's correlation coefficient, which yielded 0.875 .

To examine construct validity, exploratory factor analysis was used followed by confirmatory factor analysis. In the former, correlation coefficients were examined for the statements at first to make sure that they are in an acceptable range. Kaiser Meyer Olkin (KMO) test and Bartlett's test of sphericity were used to this end. Given that KMO $=0.858>0.7$ and that Bartlett's test was significant (Chi Square $=13500 / 19$, P-value $<0.01$ ), the presumptions for using exploratory factor analysis on TCU questionnaire with 83 statements are met. Varimax vertical rotation was employed and the factors of which the specific value was above 1 were selected for exploratory factor analysis through principle components (PC) analysis.

The primary results showed that 19 factors or elements can be used for the analysis. The total variance percentage of these 19 factors was $70.24 \%$. In addition, commonality value of each statement was high 
$(0.5<)$ so that none of the questions were omitted in this stage. Still, factor load of the rotated variables showed that some of the variables had factor loading $(0.3<)$ on two factors at the same time and therefore, they were omitted. In this way, 24 statements $(1,2,10,12,14,15,19,23,27,28,32,35,37,47$, $48,51,54,56,59,68,70,74,75$, and 81 ) were omitted. In addition, statement No.26 was omitted because of low factor loading ( $0.3>$ ) on different factors. Thus, 58 statements remained in the study. Exploratory factor analysis was repeated using the main elements of the analysis and varimax rotation. Scree plot (Diagram 1) demonstrates factor analysis is SPSS so that 13 factors or elements are fitted for the final analysis. The questions about each factor, name of each factor, and Cronbach's alpha coefficients are listed below to determine reliability of the elements. Exploratory factor analysis was completed with 11 factors and 56 statements (Table 1).

Table 1

Exploratory analysis result

\begin{tabular}{|c|c|c|c|c|}
\hline Variable & Element & $\begin{array}{l}\text { Number of } \\
\text { statements }\end{array}$ & Statements & $\begin{array}{l}\text { Cronbach's } \\
\text { alpha }\end{array}$ \\
\hline \multirow{4}{*}{$\begin{array}{l}\text { Psychological } \\
\text { performance }\end{array}$} & Self-confidence (factor No.3) & 5 & $3-4-5-6-7$ & 0.867 \\
\hline & Depression (factor No. 10) & 3 & $8-9-11$ & 0.830 \\
\hline & Anxiety (factor No.7) & 4 & $13-16-17-18$ & 0.749 \\
\hline & $\begin{array}{l}\text { Confidence in making } \\
\text { decision (factor No. 6) }\end{array}$ & 5 & $20-21-22-24-25$ & 0.908 \\
\hline \multirow[t]{4}{*}{$\begin{array}{l}\text { Social } \\
\text { performance }\end{array}$} & $\begin{array}{l}\text { Childhood problems (factor } \\
\text { no. 10) }\end{array}$ & 5 & $30-31-33-34-36$ & 0.739 \\
\hline & Hostility (factor No 11) & 7 & $\begin{array}{l}38-39-40-41-42- \\
43-44\end{array}$ & 0.941 \\
\hline & Risk taking (factor No. 8) & 4 & $45-46-49-50$ & 0.773 \\
\hline & $\begin{array}{l}\text { Social adaptability (factor } \\
\text { No. 5) }\end{array}$ & 5 & $52-53-55-57-58$ & 0.754 \\
\hline \multirow[t]{3}{*}{$\begin{array}{l}\text { Treatment } \\
\text { motivation }\end{array}$} & $\begin{array}{l}\text { Diagnosing the problem } \\
\text { (factor no.2) }\end{array}$ & 8 & $\begin{array}{l}60-61-62-63-64- \\
65-66-67\end{array}$ & 0.927 \\
\hline & $\begin{array}{l}\text { Desire to ask for help (factor } \\
\text { No.9) }\end{array}$ & 4 & $69-71-72-73$ & 0.710 \\
\hline & $\begin{array}{l}\text { Readiness for treatment } \\
\text { (factor No.4) }\end{array}$ & 6 & $\begin{array}{l}77-78-79-80-82- \\
83\end{array}$ & 0.887 \\
\hline
\end{tabular}


Table 2

Confirmatory factor analysis

\begin{tabular}{|llllll|}
\hline$\chi 2 / d f$ & RMSEA & NNFI & CFI & AGFI & Area \\
\hline $1 / 83$ & $0 / 059$ & $0 / 95$ & $0 / 95$ & $0 / 84$ & Psychological performance \\
\hline $2 / 68$ & $0 / 075$ & $0 / 91$ & $0 / 91$ & $0 / 81$ & Social performance \\
\hline $2 / 34$ & $0 / 072$ & $0 / 92$ & $0 / 92$ & $0 / 82$ & Treatment motivation \\
\hline
\end{tabular}

First and second order confirmatory factor analysis was used to examine construct validity of the tool. Only the statement No. 46 had a low factor load $(t=0.26)$ and eliminated.

\section{Analysis of the relationships}

As listed in Table 3, the variables gender, job, income, and marital status had a relationship with the psychological performance of the drug users $(P<0.05)$. However, education level, domicile, the way of using drugs, and the type of drugs did not have a significant relationship with one's social performance $(P>0.05)$. 
Table 3

Comparison of the mean score and SD of the aspects of psychological performance in terms of the demographical variables

\begin{tabular}{|c|c|c|c|c|c|c|}
\hline Variable & & $\begin{array}{l}\text { Self } \\
\text { confidence }\end{array}$ & Depression & Anxiety & $\begin{array}{l}\text { Confidence in } \\
\text { making } \\
\text { decision }\end{array}$ & $\begin{array}{l}\text { Psychological } \\
\text { performance }\end{array}$ \\
\hline \multirow[t]{3}{*}{ Gender } & $M$ & $2.0 \pm 25.95$ & $2.1 \pm 01.09$ & $\begin{array}{l}1.0 \pm \\
78.88\end{array}$ & $1.0 \pm 94.85$ & $2.0 \pm 0.64$ \\
\hline & $\mathrm{F}$ & $2.1 \pm 54.02$ & $2.0 \pm 44.90$ & $\begin{array}{l}2.0 \pm \\
7.91\end{array}$ & $1.0 \pm 99.97$ & $2.0 \pm 26.63$ \\
\hline & Sig. & 0.110 & 0.015 & 0.081 & 0.758 & 0.030 \\
\hline \multirow[t]{4}{*}{ Education } & $\begin{array}{l}\text { No high } \\
\text { school } \\
\text { diploma }\end{array}$ & $2.1 \pm 30.02$ & $2.1 \pm 1.02$ & $\begin{array}{l}1.0 \pm \\
80.88\end{array}$ & $1.0 \pm 80.85$ & $1.0 \pm 98.67$ \\
\hline & Diploma & $2.0 \pm 25.95$ & $2.1 \pm 17.18$ & $\begin{array}{l}1.0 \pm \\
88.95\end{array}$ & $1.0 \pm 96.90$ & $2.0 \pm 7.68$ \\
\hline & $\begin{array}{l}\text { College } \\
\text { degrees }\end{array}$ & $2.0 \pm 33.91$ & $2.1 \pm 1.00$ & $\begin{array}{l}1 \pm \\
76.79\end{array}$ & $2.0 \pm 22.80$ & $2.0 \pm 8.53$ \\
\hline & Sig. & 0.887 & 0.537 & 0.717 & 0.015 & 0.520 \\
\hline \multirow[t]{3}{*}{ Domicile } & City & $2.0 \pm 28.98$ & $2.1 \pm 16.07$ & $\begin{array}{l}2.0 \pm \\
5.88\end{array}$ & $1.0 \pm 94.88$ & $2.0 \pm 2.65$ \\
\hline & Rural area & $2.0 \pm 33.78$ & $2.1 \pm 6.10$ & $\begin{array}{l}1.0 \pm \\
80.92\end{array}$ & $2.0 \pm 0.75$ & $2.0 \pm 13.54$ \\
\hline & Sig. & 0.791 & 0.700 & 0.245 & 0.736 & 0.375 \\
\hline \multirow[t]{7}{*}{ Job } & $\begin{array}{l}\text { Office } \\
\text { employee }\end{array}$ & $2.0 \pm 37.95$ & $1.1 \pm 99.12$ & $\begin{array}{l}1.0 \pm \\
79.89\end{array}$ & $2.0 \pm 7.94$ & $2 \pm 5.66$ \\
\hline & Housewife & $2 \pm 92.92$ & $2 \pm 71.82$ & $\begin{array}{l}2.0 \pm \\
31.92\end{array}$ & $1.0 \pm 77.96$ & $2.0 \pm 43.65$ \\
\hline & Worker & $2.0 \pm 60.82$ & $2.0 \pm 31.98$ & $\begin{array}{l}1.0 \pm \\
93.80\end{array}$ & $1.0 \pm 77.77$ & $2.0 \pm 15.53$ \\
\hline & Freelancer & $2.0 \pm 7.91$ & $1.1 \pm 88.07$ & $\begin{array}{l}1.0 \pm \\
77.91\end{array}$ & $2.0 \pm 5.82$ & $1.0 \pm 94.63$ \\
\hline & Unemployed & $1.1 \pm 88.31$ & $2.1 \pm 16.29$ & $\begin{array}{l}1.0 \pm \\
41.75\end{array}$ & $1.1 \pm 66.07$ & $1.0 \pm 78.76$ \\
\hline & Retired & $1.0 \pm 48.36$ & $1.0 \pm 28.89$ & $\begin{array}{l}1.0 \pm \\
20.60\end{array}$ & $2.0 \pm 8.76$ & $1.0 \pm 51.56$ \\
\hline & $\begin{array}{l}\text { University } \\
\text { student }\end{array}$ & $2.0 \pm 90.14$ & $2.0 \pm 17.24$ & $\begin{array}{l}2.0 \pm \\
38.18\end{array}$ & $2.0 \pm 0.05$ & $2.0 \pm 36.1$ \\
\hline
\end{tabular}




\begin{tabular}{|c|c|c|c|c|c|c|}
\hline \multicolumn{2}{|l|}{ Variable } & \multirow{2}{*}{$\begin{array}{l}\text { Self } \\
\text { confidence } \\
0.000\end{array}$} & \multirow{2}{*}{$\begin{array}{l}\text { Depression } \\
0.009\end{array}$} & \multirow{2}{*}{$\begin{array}{l}\text { Anxiety } \\
0.023\end{array}$} & \multirow{2}{*}{ 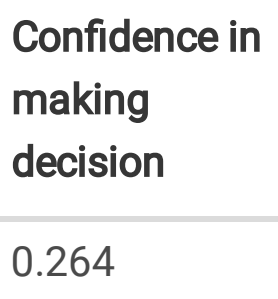 } & \multirow{2}{*}{ 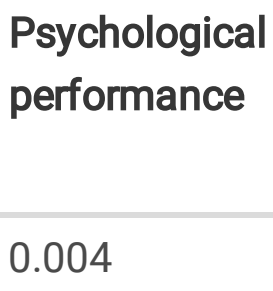 } \\
\hline & Sig. & & & & & \\
\hline \multirow[t]{5}{*}{$\begin{array}{l}\text { Marital } \\
\text { status }\end{array}$} & Unmarried & $2.0 \pm 44.93$ & $2.1 \pm 29.12$ & $\begin{array}{l}1.0 \pm \\
98.84\end{array}$ & $1.0 \pm 92.84$ & $2.0 \pm 16.62$ \\
\hline & Married & $2.0 \pm 22.91$ & $1.1 \pm 87.03$ & $\begin{array}{l}1.0 \pm \\
73.88\end{array}$ & $2.0 \pm 2.90$ & $1.0 \pm 96.61$ \\
\hline & Widower & $2.1 \pm 61.13$ & $2.1 \pm 41.14$ & $\begin{array}{l}2.1 \pm \\
0.01\end{array}$ & $1.0 \pm 80.71$ & $2.0 \pm 20.76$ \\
\hline & Widow & $1.0 \pm 31.78$ & $2.0 \pm 12.70$ & $\begin{array}{l}1.0 \pm \\
36.55\end{array}$ & $1.0 \pm 58.95$ & $1.0 \pm 59.59$ \\
\hline & Sig. & 0.001 & 0.013 & 0.046 & 0.276 & 0.008 \\
\hline \multirow[t]{4}{*}{ Income } & Low & $1.0 \pm 48.89$ & $2.1 \pm 28.02$ & $\begin{array}{l}1.0 \pm \\
95.85\end{array}$ & $1.0 \pm 96.82$ & $1.0 \pm 91.59$ \\
\hline & Average & $1.1 \pm 99.04$ & $1.1 \pm 74.10$ & $\begin{array}{l}1.0 \pm \\
61.91\end{array}$ & $1.0 \pm 83.94$ & $1.0 \pm 79.70$ \\
\hline & High & $1.0 \pm 82.96$ & $1.0 \pm 52.98$ & $\begin{array}{l}1.0 \pm \\
51.92\end{array}$ & $2.0 \pm 36.84$ & $1.0 \pm 80.61$ \\
\hline & Sig. & 0.000 & 0.000 & 0.007 & 0.053 & 0.000 \\
\hline \multirow[t]{6}{*}{$\begin{array}{l}\text { Way of } \\
\text { using }\end{array}$} & Smoking & $2.1 \pm 14.10$ & $1.1 \pm 92.07$ & $\begin{array}{l}1.0 \pm \\
83.95\end{array}$ & $1.0 \pm 94.89$ & $1.0 \pm 96.75$ \\
\hline & Injection & $2.0 \pm 70.93$ & $2.0 \pm 58.94$ & $\begin{array}{l}2.0 \pm \\
9.57\end{array}$ & $1.0 \pm 67.38$ & $2.0 \pm 26.54$ \\
\hline & Oral & $2.0 \pm 29.97$ & $1.1 \pm 98.16$ & $\begin{array}{l}1.0 \pm \\
69.75\end{array}$ & $2.0 \pm 23.92$ & $2.0 \pm 5.58$ \\
\hline & Inhaling & $2.0 \pm 56.88$ & $2.0 \pm 45.93$ & $\begin{array}{l}2.0 \pm \\
2.84\end{array}$ & $1.0 \pm 62.72$ & $2.0 \pm 16.53$ \\
\hline & Mixed & $2.0 \pm 33.95$ & $2.1 \pm 29.11$ & $\begin{array}{l}1.0 \pm \\
92.88\end{array}$ & $1.0 \pm 79.82$ & $2.0 \pm 8.79$ \\
\hline & Sig. & 0.759 & 0.365 & 0.691 & 0.063 & 0.821 \\
\hline \multirow[t]{3}{*}{$\begin{array}{l}\text { Type of } \\
\text { drug }\end{array}$} & Only one type & $2.0 \pm 12.76$ & $1.0 \pm 98.75$ & $\begin{array}{l}1.1 \pm \\
78.12\end{array}$ & $2.0 \pm 30.91$ & $2.0 \pm 4.93$ \\
\hline & Mixed & $2.0 \pm 3.93$ & $2.1 \pm 15.06$ & $\begin{array}{l}2.0 \pm \\
3.88\end{array}$ & $2.0 \pm 10.63$ & $2.0 \pm 7.93$ \\
\hline & Sig. & 0.367 & 0.158 & 0.143 & 0.287 & 0.542 \\
\hline
\end{tabular}


Table 4 lists the Pearson correlation coefficients to examine the relationship between demographical variables, psychological performance, and its aspects.

Table 4

The relationship between demographical variables and psychological performance

\begin{tabular}{|c|c|c|c|c|c|}
\hline Variable & $\begin{array}{l}\text { Self- } \\
\text { esteem }\end{array}$ & Depression & Anxiety & $\begin{array}{l}\text { Self- } \\
\text { confidence }\end{array}$ & $\begin{array}{l}\text { Psychological } \\
\text { performance }\end{array}$ \\
\hline Age & $-0.277^{\star \star}$ & $0.226 * *$ & $0.162^{\star}$ & -0.016 & $-0.249 \star \star$ \\
\hline Number of children & $-0.196 * \star$ & $0.198^{* *}$ & $0.250 * *$ & -0.076 & $-.0268 * \star$ \\
\hline History of using drugs & $-.0 .201 * *$ & $0.166^{\star \star}$ & $0.208^{\star *}$ & -.007 & $-0.219 * \star$ \\
\hline $\begin{array}{l}\text { Age of first experience of } \\
\text { drugs }\end{array}$ & -0.027 & -0.081 & -0.021 & -0.02 & -0.042 \\
\hline$* P<0.05 ; * * P<0.01$ & & & & & \\
\hline
\end{tabular}

As listed in Table 4, there is a significant relationship between age, number children, and the history of using drugs and psychological performance $(P<0.01)$. In other words, with an increase in the demographical variable, a decrease in psychological performance takes place. However, there was no relationship between the age of first experience of drugs and psychological performance $(p=0.513)$. Table 5 compares mean score and SD of the aspects of social performance in terms of demographical variables. 
Table 5

Comparison of the mean score and SD of the aspects of social performance in terms of the demographical variables

\begin{tabular}{|c|c|c|c|c|c|c|}
\hline Variable & & $\begin{array}{l}\text { Childhood } \\
\text { problems }\end{array}$ & Violence & $\begin{array}{l}\text { Risk } \\
\text { taking } \\
\text { attitude }\end{array}$ & $\begin{array}{l}\text { Social } \\
\text { adaptability }\end{array}$ & $\begin{array}{l}\text { Social } \\
\text { performance }\end{array}$ \\
\hline \multirow[t]{3}{*}{ Gender } & $M$ & $1.0 \pm 58.71$ & $\begin{array}{l}1.1 \pm \\
60.10\end{array}$ & $2.0 \pm 51.84$ & $2.0 \pm 13.79$ & $1.0 \pm 95.45$ \\
\hline & $\mathrm{F}$ & $1.0 \pm 46.054$ & $\begin{array}{l}1.1 \pm \\
58.09\end{array}$ & $2.1 \pm 47.02$ & $2.0 \pm 00.82$ & $1.0 \pm 92.41$ \\
\hline & Sig. & 0.363 & 0.934 & 0.331 & 0.316 & 0.674 \\
\hline \multirow[t]{4}{*}{ Education } & $\begin{array}{l}\text { No high } \\
\text { school } \\
\text { diploma }\end{array}$ & $1.0 \pm 60.69$ & $\begin{array}{l}1.1 \pm \\
63.10\end{array}$ & $2.0 \pm 55.85$ & $2.0 \pm 17.77$ & $1.0 \pm 99.44$ \\
\hline & Diploma & $1.0 \pm 55.74$ & $\begin{array}{l}1.1 \pm \\
50.06\end{array}$ & $2.0 \pm 52.90$ & $2.0 \pm 4.84$ & $1.0 \pm 90.48$ \\
\hline & $\begin{array}{l}\text { College } \\
\text { degrees }\end{array}$ & $1.0 \pm 50.61$ & $\begin{array}{l}1.1 \pm \\
68.17\end{array}$ & $2.0 \pm 52.85$ & $2.0 \pm 10.76$ & $1.0 \pm 95.39$ \\
\hline & Sig. & 0.700 & 0.611 & 0.963 & 0.526 & 0.432 \\
\hline \multirow[t]{3}{*}{ Domicile } & City & $1.0 \pm 54.69$ & $\begin{array}{l}1.1 \pm \\
54.11\end{array}$ & $2.0 \pm 51.87$ & $2.0 \pm 11.80$ & $1.0 \pm 92.44$ \\
\hline & Rural area & $1.0 \pm 79.61$ & $\begin{array}{l}2.0 \pm \\
18.81\end{array}$ & $20 \pm 78.76$ & $2.0 \pm 13.74$ & $2.0 \pm 22.40$ \\
\hline & Sig. & 0.086 & 0.011 & 0.142 & 0.872 & 0.004 \\
\hline \multirow[t]{7}{*}{ Job } & $\begin{array}{l}\text { Office } \\
\text { employee }\end{array}$ & $1.0 \pm 47.64$ & $\begin{array}{l}1.0 \pm \\
45.94\end{array}$ & $2.0 \pm 72.77$ & $2.0 \pm 28.79$ & $1.0 \pm 98.38$ \\
\hline & Housewife & 1.0.50.60 & $\begin{array}{l}1.1 \pm \\
65.12\end{array}$ & $2.0 \pm 73.84$ & $1.0 \pm 85.72$ & $1.0 \pm 93.35$ \\
\hline & Worker & $1.0 \pm 74.74$ & $\begin{array}{l}1.1 \pm \\
86.18\end{array}$ & $2.0 \pm 48.77$ & $2.0 \pm 07.73$ & $2.0 \pm 4.50$ \\
\hline & Freelancer & $1.0 \pm 55.69$ & $\begin{array}{l}1.1 \pm \\
56.09\end{array}$ & $2.0 \pm 54.87$ & $2.0 \pm 16.83$ & $1.0 \pm 95.44$ \\
\hline & Unemployed & $1.0 \pm 40.77$ & $\begin{array}{l}1.1 \pm \\
56.34\end{array}$ & $2.1 \pm 4.07$ & $1.0 \pm 82.84$ & $1.0 \pm 70.48$ \\
\hline & Retired & $0.0 \pm 94.43$ & $\begin{array}{l}0.0 \pm \\
60.60\end{array}$ & $1.1 \pm 93.14$ & $1.0 \pm 92.67$ & $1.0 \pm 35.29$ \\
\hline & $\begin{array}{l}\text { University } \\
\text { student }\end{array}$ & $2.0 \pm 10.14$ & $\begin{array}{l}2.0 \pm \\
14.20\end{array}$ & $2.0 \pm 67.94$ & $1.0 \pm 80.29$ & $2.0 \pm 18.15$ \\
\hline
\end{tabular}




\begin{tabular}{|c|c|c|c|c|c|c|}
\hline \multicolumn{2}{|l|}{ Variable } & \multirow{2}{*}{$\begin{array}{l}\text { Childhood } \\
\text { problems }\end{array}$} & \multirow{2}{*}{$\begin{array}{l}\text { Violence } \\
0.191\end{array}$} & \multirow{2}{*}{$\begin{array}{l}\text { Risk } \\
\text { taking } \\
\text { attitude }\end{array}$} & \multirow{2}{*}{ 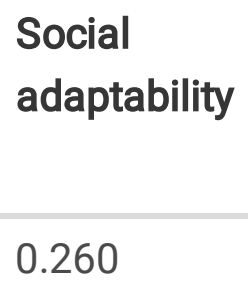 } & \multirow{2}{*}{$\begin{array}{l}\begin{array}{l}\text { Social } \\
\text { performance }\end{array} \\
0.012\end{array}$} \\
\hline & Sig. & & & & & \\
\hline \multirow[t]{5}{*}{$\begin{array}{l}\text { Marital } \\
\text { status }\end{array}$} & Unmarried & $1.0 \pm 65.71$ & $\begin{array}{l}1.1 \pm \\
74.10\end{array}$ & $2.0 \pm 43.91$ & $2.0 \pm 00.75$ & $1.0 \pm 96.42$ \\
\hline & Married & $1.0 \pm 54.67$ & $\begin{array}{l}1.1 \pm \\
47.06\end{array}$ & $2.0 \pm 70.79$ & $2.0 \pm 28.77$ & $2.0 \pm 00.43$ \\
\hline & Widower & $1.0 \pm 57.77$ & $\begin{array}{l}1.1 \pm \\
82.28\end{array}$ & $2.0 \pm 30.90$ & $1.0 \pm 81.82$ & $1.0 \pm 88.47$ \\
\hline & Widow & $1.0 \pm 20.55$ & $\begin{array}{l}1.0 \pm \\
30.93\end{array}$ & $1.0 \pm 78.89$ & $1.0 \pm 47.73$ & $1.0 \pm 44.44$ \\
\hline & Sig. & 0.224 & 0.121 & 0.001 & 0.000 & 0.001 \\
\hline \multirow[t]{4}{*}{ Income } & Low & $1.0 \pm 64.73$ & $\begin{array}{l}1.1 \pm \\
76.10\end{array}$ & $2.0 \pm 50.86$ & $2.0 \pm 5.74$ & $1.0 \pm 98.45$ \\
\hline & Average & $1.0 \pm 49.62$ & $\begin{array}{l}1.1 \pm \\
46.04\end{array}$ & $2.0 \pm 56.88$ & $2.0 \pm 11.87$ & $1.0 \pm 91.44$ \\
\hline & High & $1.0 \pm 17.44$ & $\begin{array}{l}0.0 \pm \\
76.92\end{array}$ & $2.0 \pm 73.80$ & $2.0 \pm 60.79$ & $1.0 \pm 82.38$ \\
\hline & Sig. & 0.010 & 0.000 & 0.484 & 0.013 & 0.0478 \\
\hline \multirow[t]{6}{*}{$\begin{array}{l}\text { Way of } \\
\text { using }\end{array}$} & Smoking & $1.0 \pm 53.70$ & $\begin{array}{l}1.1 \pm \\
59.07\end{array}$ & $2.0 \pm 54.90$ & $2.0 \pm 00.80$ & $1.0 \pm 91.49$ \\
\hline & Injection & $2.0 \pm 40.73$ & $\begin{array}{l}2.1 \pm \\
45.00\end{array}$ & $1.0 \pm 78.87$ & $1.0 \pm 70.76$ & $2.0 \pm 0.8 .37$ \\
\hline & Oral & $1.0 \pm 36.58$ & $\begin{array}{l}1.1 \pm \\
22.12\end{array}$ & $2.0 \pm 90.79$ & $2.0 \pm 49.83$ & $1.0 \pm 99.37$ \\
\hline & Inhaling & $1.0 \pm 91.73$ & $\begin{array}{l}2.1 \pm \\
38.00\end{array}$ & $2.0 \pm 09.92$ & $1.0 \pm 98.58$ & $2.0 \pm 08.62$ \\
\hline & Mixed & $1.0 \pm 70.71$ & $\begin{array}{l}1.1 \pm \\
95.02\end{array}$ & $2.0 \pm 33.66$ & $2.0 \pm 07.94$ & $2.0 \pm 01.45$ \\
\hline & Sig. & 0.074 & 0.084 & 0.012 & 0.121 & 0.431 \\
\hline \multirow[t]{3}{*}{$\begin{array}{l}\text { Type of } \\
\text { drug }\end{array}$} & Only one type & $1.0 \pm 68.75$ & $\begin{array}{l}1.1 \pm \\
86.11\end{array}$ & $2.0 \pm 63.89$ & $1.0 \pm 70.78$ & $1.0 \pm 96.49$ \\
\hline & Mixed & $1.0 \pm 75.59$ & $\begin{array}{l}1.0 \pm \\
80.87\end{array}$ & $2.1 \pm 66.02$ & $1.0 \pm 49.69$ & $1.0 \pm 92.41$ \\
\hline & Sig. & 0.42 & 0.538 & 0.654 & 0.041 & 0.739 \\
\hline
\end{tabular}


As the findings showed, domicile, job, income, and marital status had a relationship with social performance of the patients $(P<0.05)$. However, gender $(p=0.674)$, education level $(p=0.432)$, way of using drug $(p=0.431)$, and type of drug $(p=0.739)$ did not have a significant relationship with social performance. Table 6 lists Pearson correlation coefficients for the relationship between demographical variables, psychological performance, and its aspects.

Table 6

The relationship between demographical variables and psychological performance

\begin{tabular}{|llllll|}
\hline Variable & $\begin{array}{l}\text { Childhood } \\
\text { problems }\end{array}$ & Violence & $\begin{array}{l}\text { Risk taking } \\
\text { attitude }\end{array}$ & $\begin{array}{l}\text { Social } \\
\text { adaptability }\end{array}$ & $\begin{array}{l}\text { Social } \\
\text { performance }\end{array}$ \\
\hline Age & -0.084 & $0.143^{*}$ & 0.060 & 0.089 & -0.052 \\
\hline Number of children & -.098 & -.097 & 0.002 & 0.067 & -0.067 \\
\hline History of using drugs & -0.064 & -0.093 & -.0 .012 & 0.028 & -0.075 \\
\hline $\begin{array}{l}\text { Age of first experience } \\
\text { of drugs }\end{array}$ & -0.040 & 0.036 & 0.074 & 0.071 & 0.030 \\
\hline$* P<0.05 ; * * P<0.01$ & & & & & \\
\hline
\end{tabular}

As listed in Table 6, there is a negative significant relationship between age and violence in the patients $(p<0.01)$. In other words, with an increase in age, violence declines in the patients. There was no significant relationship between other demographical variables and social performance $(p>0.05)$. As listed in Table 7, none of the demographical variables are related to the motivation for treatment in the subjects $(p>0.5)$. Only marital status was significantly related to readiness for treatment. So that, the widowers/widows had more motivation to quit. In addition, the type of drug has a significant relationship with readiness for treatment $(P>0.05)$; so that patients who use only one type of drug have more desire for treatment. 
Table 7

- Comparing mean score and SD of the aspects of motivation in terms of demographical variables.

\begin{tabular}{|c|c|c|c|c|c|}
\hline Variable & & $\begin{array}{l}\text { Recognizing the } \\
\text { problem }\end{array}$ & $\begin{array}{l}\text { Desire to } \\
\text { seek help }\end{array}$ & $\begin{array}{l}\text { Readiness for } \\
\text { treatment }\end{array}$ & $\begin{array}{l}\text { Treatment } \\
\text { motivation }\end{array}$ \\
\hline \multirow[t]{3}{*}{ Gender } & $M$ & $2.0 \pm 96.87$ & $2.0 \pm 49.70$ & $2.0 \pm 37.95$ & $2.0 \pm 60.66$ \\
\hline & $\mathrm{F}$ & $2.0 \pm 89.80$ & $2.0 \pm 40.62$ & $2.0 \pm 54.85$ & $2.0 \pm 61.65$ \\
\hline & Sig. & 0.679 & 0.442 & 0.285 & 0.957 \\
\hline \multirow[t]{4}{*}{ Education } & $\begin{array}{l}\text { No high school } \\
\text { diploma }\end{array}$ & $2.0 \pm 86.84$ & $2.1 \pm 39.02$ & $2.0 \pm 36.93$ & $2.0 \pm 54.66$ \\
\hline & Diploma & $3.0 \pm 4.85$ & $2.1 \pm 62.18$ & $2.0 \pm 35.99$ & $2.0 \pm 67.64$ \\
\hline & College degrees & $2.0 \pm 97.92$ & $2.1 \pm 40.00$ & $2.0 \pm 53.84$ & $2.0 \pm 63.70$ \\
\hline & Sig. & 0.331 & 0.056 & 0.506 & 0.367 \\
\hline \multirow[t]{3}{*}{ Domicile } & City & $2.0 \pm 97.85$ & $2.1 \pm 46.07$ & $2.0 \pm 39.94$ & $2.0 \pm 60.67$ \\
\hline & Rural area & $2.0 \pm 75.94$ & $2.1 \pm 62.10$ & $2.0 \pm 43.88$ & $2.0 \pm 60.57$ \\
\hline & Sig. & 0.321 & 0.260 & 0.839 & 0.967 \\
\hline \multirow[t]{8}{*}{ Job } & $\begin{array}{l}\text { Office } \\
\text { employee }\end{array}$ & $2.0 \pm 98.97$ & $2.0 \pm 45.79$ & $2.0 \pm 43.96$ & $2.0 \pm 62.78$ \\
\hline & Housewife & $3.0 \pm 6.64$ & $2.0 \pm 45.53$ & $2.0 \pm 63.85$ & $2.0 \pm 71.58$ \\
\hline & Worker & $2.0 \pm 90.83$ & $2.0 \pm 50.76$ & $2.0 \pm 49.89$ & $2.0 \pm 63.69$ \\
\hline & Freelancer & $2.0 \pm 91.91$ & $2.0 \pm 49.65$ & $2.0 \pm 34.94$ & $2.0 \pm 58.64$ \\
\hline & Unemployed & $3.0 \pm 16.71$ & $2.0 \pm 51.65$ & $2.1 \pm 24.11$ & $2.0 \pm 64.23$ \\
\hline & Retired & $2.0 \pm 50.40$ & $2.0 \pm 35.70$ & $1.0 \pm 77.98$ & $2.0 \pm 21.58$ \\
\hline & $\begin{array}{l}\text { University } \\
\text { student }\end{array}$ & $3.0 \pm 50.35$ & $2.1 \pm 0.06$ & $2.0 \pm 33.71$ & $2.0 \pm 61.71$ \\
\hline & Sig. & 0.682 & 0.967 & 0.548 & 0.847 \\
\hline \multirow{5}{*}{$\begin{array}{l}\text { Marital } \\
\text { status }\end{array}$} & Unmarried & $3.0 \pm 4.84$ & $2.0 \pm 56.68$ & $2.0 \pm 42.95$ & $2.0 \pm 68.65$ \\
\hline & Married & $2.0 \pm 90.86$ & $2.0 \pm 47.67$ & $2.0 \pm 41.90$ & $2.0 \pm 60.65$ \\
\hline & Widower & $3.0 \pm 6.96$ & $2.0 \pm 28.75$ & $2.0 \pm 56.88$ & $2.0 \pm 63.74$ \\
\hline & Widow & $2.0 \pm 56.91$ & $2.0 \pm 50.77$ & $1.1 \pm 44.04$ & $2.0 \pm 17.60$ \\
\hline & Sig. & 0.262 & 0.320 & 0.005 & 0.122 \\
\hline Income & Low & $2.0 \pm 98.83$ & $2.0 \pm 53.65$ & $2.0 \pm 48.89$ & $2.0 \pm 66.63$ \\
\hline
\end{tabular}




\begin{tabular}{|c|c|c|c|c|c|}
\hline \multicolumn{2}{|l|}{ Variable } & \multirow{2}{*}{$\begin{array}{l}\text { Recognizing the } \\
\text { problem } \\
2.0 \pm 96.89\end{array}$} & \multirow{2}{*}{$\begin{array}{l}\begin{array}{l}\text { Desire to } \\
\text { seek help }\end{array} \\
2.0 \pm 42.76\end{array}$} & \multirow{2}{*}{$\begin{array}{l}\begin{array}{l}\text { Readiness for } \\
\text { treatment }\end{array} \\
2.1 \pm 19.02\end{array}$} & \multirow{2}{*}{$\begin{array}{l}\begin{array}{c}\text { Treatment } \\
\text { motivation }\end{array} \\
2.0 \pm 53.68\end{array}$} \\
\hline & Average & & & & \\
\hline & High & $2.0 \pm 69.99$ & $2.0 \pm 23.70$ & $2.0 \pm 36.92$ & $2.0 \pm 42.78$ \\
\hline & Sig. & 0.37 & 0.130 & 0.088 & 0.146 \\
\hline \multirow{6}{*}{$\begin{array}{l}\text { Way of } \\
\text { using }\end{array}$} & Smoking & $2.0 \pm 99.90$ & $2.0 \pm 51.74$ & $2.1 \pm 37.03$ & $2.0 \pm 62.69$ \\
\hline & Injection & $3.0 \pm 2.81$ & $2.0 \pm 56.46$ & $2.0 \pm 75.65$ & $2.0 \pm 78.49$ \\
\hline & Oral & $3.0 \pm 3.89$ & $2.0 \pm 42.70$ & $2.0 \pm 54.91$ & $2.0 \pm 66.66$ \\
\hline & Inhaling & $3.0 \pm 0.69$ & $20 \pm 59.74$ & $2 \pm 86.67$ & $2.0 \pm 82.59$ \\
\hline & Mixed & $2.0 \pm 95.95$ & $2.1 \pm 50.11$ & $2.0 \pm 33.99$ & $2.0 \pm 59.92$ \\
\hline & Sig. & 0.942 & 0.980 & 0.275 & 0.696 \\
\hline \multirow{3}{*}{$\begin{array}{l}\text { Type of } \\
\text { drug }\end{array}$} & Only one type & $20 \pm 78.76$ & $3.0 \pm 1.70$ & $2.0 \pm 35.88$ & $2.0 \pm 71.74$ \\
\hline & Mixed & $3.0 \pm 10.93$ & $2.0 \pm 89.98$ & $2.0 \pm 3.83$ & $20 \pm 67.69$ \\
\hline & Sig. & 0.087 & 0.158 & 0.049 & 0.542 \\
\hline
\end{tabular}

As listed in Table 8, there is a negative relationship between number of children and motivation for treatment $(p<0.05, r=-0.139)$. That is, with an increase in the number of children, the motivation in patients declines.

Table 8

The relationship between demographical variables and motivation

\begin{tabular}{|lllll|}
\hline Variable & $\begin{array}{l}\text { Recognizing the } \\
\text { problem }\end{array}$ & $\begin{array}{l}\text { Desire to } \\
\text { seek help }\end{array}$ & $\begin{array}{l}\text { Readiness for } \\
\text { treatment }\end{array}$ & $\begin{array}{l}\text { Treatment } \\
\text { motivation }\end{array}$ \\
\hline Age & -0.067 & -0.080 & -0.037 & -0.019 \\
\hline Number of children & $-0.130 *$ & -0.075 & -0.120 & $-0.139 *$ \\
\hline History of using drugs & -0.110 & -0.055 & -0.085 & -0.107 \\
\hline $\begin{array}{l}\text { Age of first experience } \\
\text { of drugs }\end{array}$ & -0.004 & $0.130 *$ & 0.042 & 0.63 \\
\hline$* P<0.05, * * P<0.01$ & & & & \\
\hline
\end{tabular}

\section{Discussion}


Perceiving the effects of demographical variables on psychological, social, and motivational performance of drug users may lead to better treatment protocols. The relationship between the demographical variables of drug users and their social, psychological, and motivational performance was examined. Gender was effective in depression score of the participants - i.e. an aspect of psychological performance- so that it was higher in women than men. In general, gender was effective in psychological performance of drug users so that female drug addicts, being more sensitive than men, were more vulnerable to psychological damages. This finding was more consistent with other studies $(9,21)$. However, the effect of gender on social performance of the drug users was not significant.

Education level of the participants was effective in their confidence in decision making -i.e. an aspect of psychological performance. In general, however, the effect of education level on psychological and social performance was not significant. Domicile was another variable under study and it was effective in the level of violent behavior -i.e. an aspect of social performance. That is, city dwellers were less violent than those living in rural areas. One probable reason for this is that the latter group live in a smaller community and they feel more pressure by their society for being a drug addict. In general, and consistent with Poudel et al. (2016) (22), the small sample group of rural dwellers and the considerable level of interactions between villages and cities in Iran can explain this finding.

Job was another effective factor in psychological and social performance. That is, those who had a job had a better social and psychological performance than those without a job. The results showed that job was effective in the participants' self-esteem, depression, and anxiety (psychological performance) and risk-taking attitudes (social performance). This is consistent with other studies $(3,9)$. Many studies have shown that having a decent job is an effective factor in enabling the care-seekers (21) and it can improve their physical and psychological performance $(3,5,13,21)$.

Marital status was effective in self-esteem, depression, and anxiety (psychological performance); so that the unmarried individuals had a better psychological performance. This finding is consistent with other studies like $(9,14)$. Risk-taking attitude and social adaptability in the married individual was higher than the others; which is consistent with Gyawali and Sarkar (2016) (23).

Individuals with a higher income had higher self-esteem and confidence in decision making. They also had lower anxiety and depression (psychological performance). Moreover, drug users with higher income had a fewer childhood problems, were less violent, and were more socially adaptable. To explain this, a better economic condition attenuates social problems and improves the quality of life (15). Socioeconomic condition of family and proper family support (14) can be effective in self-esteem, happiness(24), and even the quality of life (16) as they play a key role in treatment and prevention of relapse (9).

There was a negative relationship between age and psychological performance of care-seekers. That is, the older individuals had more psychological performance problems comparing with the younger subjects. In addition, the level of violence was lower in the older drug users. Number of children and the history of using drugs were of other variables effective in psychological performance of the subjects. 
These two variables had a negative relationship with psychological performance of the participants. Drug users with more children or a longer history of suing drugs had a lower psychological performance. These findings are consistent with Poudel et al. (2016) (22).

Demographical variables did not have a notable effect on motivation for treatment. Only marital status had a significant relationship with readiness for treatment; that is, unmarried individuals had more motivation for treatment. One probable reason is that unmarried addicts might have higher hopes for starting a new life. This finding is consistent with German et al. (2006) (17). Another explanation for this might be the fact that married drug users have to spend more time and money on the welfare of their children as the first priority of the family. In addition, desire for treatment was higher in the subjects who only used one type of drug; which is consistent with Targowski et al. (2004) (16). Another reason for this finding is that drug users who only use one type drug have a higher hope for rehabilitation. It appears, however, that the demographical variables are not very effective in the motivation for treatment. Probably, other factors including inner, personal, and family factors are more effective in the motivation for treatment.

\section{Conclusion}

The result showed that demographical variables were effective in the process of treatment and rehabilitation of drug users' care-seekers. These variables were related to psychological, social, and motivational performance of the care-seekers. Therefore, the demographical variables need to be taken into account in therapeutic and rehabilitation planning.

\section{Abbreviations}

Content Validity Index (CVI)

Content Validity Ratio (CVR)

Kaiser Meyer Olkin (KMO)

Texas Cristian University (TCU)

Principle Components (PC)

Kermanshah University of Medical Sciences (KUMS)

\section{Declarations}

\section{Ethical considerations}

After issuing the ID code (IR.KUMS.REC.1396.749) by the KUMS Ethics Committee, data collection was initiated. First, the participants were provided with some explanations on the study and its objectives. All 
participants completed written consent to participate in the study.

\section{Consent to publication:}

Not applicable.

\section{Availability of data and materials}

The datasets used and analyzed during the current study are available from the corresponding author on reasonable request.

\section{Competing interests:}

The authors declare that they have no conflict of interest about this work.

\section{Funding:}

This study was drawn from a research project (No. 97037) sponsored by deputy of research and technology of KUMS. The cost of the payment is spent on the design and implementation of the study

\section{Author's contribution}

S Sh, contributed in study concept, study design, data collection and manuscript preparation.

A J, contributed in study concept, study design, data Analysis, manuscript preparation and submitting the manuscript

$\mathbf{R} \mathbf{J}$, contributed in study concept, study design and manuscript preparation.

A Gh, contributed in study design, data analysis and manuscript preparation.

\section{Acknowledgement}

We are grateful to the Deputy for Research and Technology, Kermanshah University of Medical Sciences, for cooperating in this research. We thank all the participants in the study. 


\section{References}

1. Whittaker E, Swift W, Roxburgh A, Dietze P, Cogger S, Bruno R, et al. Multiply disadvantaged: Health and service utilisation factors faced by homeless injecting drug consumers in Australia. Drug and alcohol review. 2015;34(4):379-87.DOI: 10.1111/dar.12257.

2. Komaromy M, Duhigg D, Metcalf A, Carlson C, Kalishman S, Hayes L, et al. Project ECHO (Extension for Community Healthcare Outcomes): A new model for educating primary care providers about treatment of substance use disorders. Substance abuse. 2016;37(1):20-4.DOI: 10.1080/08897077.2015.1129388.

3. Guydish J, Passalacqua E, Pagano A, Martínez C, Le T, Chun J, et al. An international systematic review of smoking prevalence in addiction treatment. Addiction. 2016;111(2):220-30.DOI: 10.1111/add.13099.

4. Urbanoski KA. Coerced addiction treatment: Client perspectives and the implications of their neglect. Harm Reduction Journal. 2010;7(1):13.DOI: 10.1186/1477-7517-7-13.

5. Tavakoli Ghouchani H, Niknami S, Aminshokravi F, Hojjat SK. Factors Related to Addiction Treatment Motivations; Validity and Reliability of an Instrument. Journal of Research in Health Sciences. 2016;16(3):147-52.

6. Shahbazi F, Mirtorabi SD, Ghadirzadeh MR, Hashemi-Nazari SS, Barzegar A. Characterizing Mortality from Substance Abuse in Iran: An Epidemiological Study during March 2014 to February 2015. Addict Health. 2017;9(3):166-74.

7. Alitabar S, Falahatpisheh M, Asgarabad M, Arvin M, Sarvestani A. Psychometric Properties of the Problems Assessment for Substance Using Psychiatric Patients. Community Health. 2016;3(1):1120.(inpersian).

8. Heidari J, Jafari H, Hoseini S, Jannati Y, Mohammadpoor R, Mahmoodi G. Study of Psychosocial Status of Addicts in Sari. Journal of Mazandaran University of Medical Sciences. 2006;16(52):10916.

9. Scorzelli JF, Chaudhry SZ. Relationship between anxiety and addiction to a depressant drug. Journal of Psychoactive Drugs. 2009;41(1):61-6.DOI: 10.1080/02791072.2009.10400675.

10. Jalali A, Hassanpor-Dehkordi A, Mahvar T, Moradi M, Dinmohammadi M. Psychological needs of men under methadone maintenance treatment: A mixed method study. Heroin Addiction and Related Clinical Problems. 2015;17:23-31.

11. Rahimi S, Jalali A, Jalali R. Psychological Needs of Women Treated with Methadone: Mixed Method Study. Alcoholism Treatment Quarterly. 2019;37(3):328-41.DOI: 10.1080/07347324.2018.1554982.

12. Sau M, Mukherjee A, Manna N, Sanyal S. Sociodemographic and substance use correlates of repeated relapse among patients presenting for relapse treatment at an addiction treatment center in Kolkata, India. Afr Health Sci. 2013;13(3):791-9.DOI: 10.4314/ahs.v13i3.39. 
13. Simpson D, GW J. Motivation as a predictor of early dropout from drug abuse treatment, Psychotherapy. Theory, Research, Practice, Training. 1993;30(2):357.DOI: 10.1037/00333204.30.2.357.

14. West R, McEwen A, Bolling K, L. O. Smoking cessation and smoking patterns in the general population: a 1-year follow-up. Addiction Research. 2001;96(6):891-902.DOI: 10.1046/j.13600443.2001.96689110.x.

15. Battjes RJ, Gordon MS, O'Grady KE, Kinlock TW, Carswell MA. Factors that predict adolescent motivation for substance abuse treatment, Journal of substance abuse treatment. Journal of Substance Abuse Treatment. 2003;24(3):221-32.DOI: 10.1016/s0740-5472(03)00022-9.

16. Targowski T, Grabowska P, Rozyńska R, From S, Mierzejewska J, Niedziałkowski P. Comparative analysis of nicotin dependence and motivating to quit smoking in patients with bronchial asthma or copd. przegl lek. 2004;61(10):1046-8.

17. German D, Sherman SG, Sirirojn B, Thomson N, Aramrattana A, Celentano DD. Motivations for methamphetamine cessation among young people in northern Thailand. Addiction. 2006;101(8):1143-52.DOI: 10.1111/j.1360-0443.2006.01490.x.

18. Behravan H, Miranvari S. A Sociological Analysis of Drug Addict's Relapse in the in the center of the therapeutic community (TC) in Mashhad. Iranian Journal of Social Problems. 2010;1(1):45-78. (Inpersian).

19. Wild D, Grove A, Martin M, Eremenco S, McElroy S, Verjee-Lorenz A, et al. Principles of Good Practice for the Translation and Cultural Adaptation Process for Patient-Reported Outcomes (PRO) Measures: report of the ISPOR Task Force for Translation and Cultural Adaptation. Value in health : the journal of the International Society for Pharmacoeconomics and Outcomes Research. 2005;8(2):94-104.DOI: 10.1111/j.1524-4733.2005.04054.x.

20. Knight K, Holcom M, Simpson DD. TCU psychosocial functioning and motivation scales: Manual on psychometric properties. Fort worth: texas christian university, institute of behavioral research. 1994.

21. Kalivas PW, Volkow ND. The neural basis of addiction: a pathology of motivation and choice. American Journal of Psychiatry. 2005;162(8):1403-13.DOI: 10.1176/appi.ajp.162.8.1403.

22. Poudel A, Sharma C, Gautam S, Poudel A. Psychosocial problems among individuals with substance use disorders in drug rehabilitation centers, Nepal. Subst Abuse Treat Prev Policy. 2016;11(1):28-.DOI: 10.1186/s13011-016-0072-3.

23. Gyawali S, Sarkar S. Tramadol for maintenance treatment for an elderly "doda" (poppy husk) user. Journal of Geriatric Mental Health. 2016;3(2):179-81.DOI: 10.4103/2348-9995.195682.

24. Jalali A, Behrouzi MK, Salari N, Bazrafshan MR, Rahmati M. The Effectiveness of Group Spiritual Intervention on Self-esteem and Happiness among Men Undergoing Methadone Maintenance Treatment. Current drug abuse reviews. 2018.DOI: 10.2174/1874473711666180510164420. 